\title{
On the Fading Number of Multi-Antenna Systems over Flat Fading Channels with Memory and Incomplete Side Information
}

\author{
Amos Lapidoth Stefan M. Moser \\ Signal and Information Processing Laboratory \\ Swiss Federal Institute of Technology (ETH) Zurich \\ CH-8092 Zurich, Switzerland \\ e-mail: $\{$ lapidoth,moser\}@isi.ee.ethz.ch
}

\begin{abstract}
Upper and lower bounds are derived on the capacity and fading number of multi-antenna systems operating over flat fading channels. The fading process may exhibit memory, and the receiver is assumed to have access to some incomplete (possibly null) side information related to the fading realization. For single-input single-output (SISO) systems these bounds typically yield an exact expression for the system's fading number. This expression is evaluated explicitly in the SISO Gaussian case as a function of the fading mean, its power spectral density, and the mutual information rate between the fading process and side information. In the absence of side information, the fading number of such systems depends only on the mean and variance of the fading process and on the minimum mean squared-error in predicting the present fading from the past; it is not directly related to the Doppler spread or coherence time.
\end{abstract}

We consider a channel with $n_{\mathrm{T}}$ transmit antennae and $n_{\mathrm{R}}$ receive antennae whose time- $k$ output $\mathbf{Y}_{k} \in \mathbb{C}^{n_{\mathrm{R}}}$ is given by

$$
\mathbf{Y}_{k}=\mathbb{H}_{k} \mathbf{x}_{k}+\mathbf{Z}_{k}
$$

where $\mathbf{x}_{k} \in \mathbb{C}^{n_{\mathrm{T}}}$ denotes the power- $\left\|\mathbf{x}_{k}\right\|^{2}$ time- $k$ input vector, the vectors $\left\{\mathbf{Z}_{k}\right\}$ are spatially and temporally white zero-mean circularly-symmetric Gaussians of covariance matrix $\sigma^{2} \mathrm{I}$, and the components of the random matrices $\left\{\mathbb{H}_{k}\right\}$ are jointly stationary and ergodic stochastic processes independent of $\left\{\mathbf{Z}_{k}\right\}$. We will consider the case where the receiver (but not the transmitter) has access to some side information in the form of a random process $\left\{\mathbf{S}_{k}\right\}$, where $\left\{\left(\mathbb{H}_{k}, \mathbf{S}_{k}\right)\right\}$ is a jointly stationary and ergodic process independent of $\left\{\mathbf{Z}_{k}\right\}$. We denote by $C_{\mathrm{SI}}(\mathrm{SNR})=C_{\mathrm{SI}}\left(\mathcal{E}_{\mathrm{s}} / \sigma^{2}\right)$ the capacity of this channel with side information at the receiver under an average power constraint

$$
\mathrm{E}\left[\left\|\mathbf{X}_{k}\right\|^{2}\right] \leq \mathcal{E}_{\mathrm{s}}
$$

Extending [1, Theorem 1] to fading with memory and side information at the receiver we have:

Theorem 1. (Generalized Villa Serbelloni) Assume that the stationary and ergodic matrix-valued fading process $\left\{\mathbb{H}_{k}\right\}$ is of finite expected Frobenius norm $\mathrm{E}\left[\left\|\mathbb{H}_{k}\right\|_{\mathrm{F}}^{2}\right]<\infty$, and that the conditional differential entropy rate $h\left(\left\{\mathbb{H}_{k}\right\} \mid\left\{\mathbf{S}_{k}\right\}\right)$ is finite. Then the fading number $\chi\left(\left\{\mathbb{H}_{k}\right\} \mid\left\{\mathbf{S}_{k}\right\}\right)$ defined by

$$
\chi\left(\left\{\mathbb{H}_{k}\right\} \mid\left\{\mathbf{S}_{k}\right\}\right) \triangleq \varlimsup_{\mathrm{SNR} \uparrow \infty}\left\{C_{\mathrm{SI}}(\mathrm{SNR})-\log \log \mathrm{SNR}\right\}
$$

(see also [2]) is finite and is upper bounded by

$$
\begin{aligned}
\chi\left(\left\{\mathbb{H}_{k}\right\} \mid\left\{\mathbf{S}_{k}\right\}\right) & \leq \chi\left(\left\{\mathbb{H}_{k}\right\}\right)+I\left(\left\{\mathbb{H}_{k}\right\} ;\left\{\mathbf{S}_{k}\right\}\right) \\
& \leq \chi \mathrm{IID}+h\left(\mathbb{H}_{1}\right)-h\left(\left\{\mathbb{H}_{k}\right\} \mid\left\{\mathbf{S}_{k}\right\}\right)
\end{aligned}
$$

where $\chi\left(\left\{\mathbb{H}_{k}\right\}\right)$ denotes the fading number in the absence of side information, and $\chi_{\mathrm{IID}}$ is the fading number corresponding to memoryless fading of equal marginal distribution and without side information.

Note: The double-logarithmic behavior of channel capacity continues to hold even if $\left\{\mathbf{Z}_{k}\right\}$ is not Gaussian. It suffices that it be stationary and ergodic with finite second moment and finite entropy rate.

Theorem 2. For SISO systems $\left(n_{\mathrm{T}}=n_{\mathrm{R}}=1\right)$ satisfying the assumptions of Theorem 1

$$
\chi\left(\left\{H_{k}\right\} \mid\left\{S_{k}\right\}\right)=\log \pi+\mathrm{E}\left[\log \left|H_{1}\right|^{2}\right]-h\left(\left\{H_{k}\right\} \mid\left\{S_{k}\right\}\right) .
$$

Corollary 3. Consider a SISO fading process $\left\{H_{k}\right\}$ such that for some specular component $d \in \mathbb{C}$ the process $\left\{H_{k}-d\right\}$ is a zero-mean unit-variance circularly symmetric stationary and ergodic complex Gaussian process whose spectrum is of continuous part $F^{\prime}(\lambda),-1 / 2 \leq \lambda \leq 1 / 2$. Then

$$
\chi=\log |d|^{2}-\operatorname{Ei}\left(-|d|^{2}\right)-1+\log \frac{1}{\varepsilon^{2}}+I\left(\left\{H_{k}\right\} ;\left\{\mathbf{S}_{k}\right\}\right)
$$

where $\varepsilon^{2}>0$ denotes the minimum mean squared-error in predicting the present fading from its past (assumed positive)

$$
\varepsilon^{2}=\exp \left\{\int_{-1 / 2}^{1 / 2} \log F^{\prime}(\lambda) \mathrm{d} \lambda\right\}
$$

and the mutual information rate is assumed finite.

For general MISO $\left(n_{\mathrm{R}}=1\right)$ systems with memory and side information we were as of yet unable to compute the fading number. If the fading is memoryless and there is no side information, then beam-forming is asymptotically optimal and

$$
\chi=\log \pi+\sup _{\|\hat{\mathbf{x}}\|=1}\left\{\mathrm{E}\left[\log |\mathbb{H} \hat{\mathbf{x}}|^{2}\right]-h(\mathbb{H} \hat{\mathbf{x}})\right\} .
$$

For SIMO systems $\chi$ admits a simple expression even in the presence of memory and side information.

Note: While we have assumed throughout that the side information is unavailable to the transmitter, we suspect that in the SISO case the results do not depend critically on this assumption in view of the limited gains afforded by optimal power allocation in the $\log \log \mathrm{SNR}$ regime.

\section{REFERENCES}

[1] A. Lapidoth and S. Moser, "Convex-Programming Bounds on the Capacity of Flat-Fading Channels," Proc. 2001 IEEE Int. Symposium on Inform. Theory, p. 52, Washington DC, June 2001.

[2] A. Lapidoth and S. Moser, "On the Fading Number of MultiAntenna Systems," Proc. 2001 IEEE Inform. Theory Workshop, Cairns, Australia, Sept. 2001. 